\title{
Supersolid stripes enhanced by correlations in a Raman spin-orbit-coupled system
}

\author{
J. Sánchez-Baena $\odot,{ }^{*}$ J. Boronat ${ }^{\dagger}$ and F. Mazzanti ${ }^{\ddagger}$ \\ Departament de Física, Universitat Politècnica de Catalunya, Campus Nord B4-B5, E-08034 Barcelona, Spain
}

(Received 19 December 2019; revised manuscript received 9 March 2020; accepted 10 March 2020; published 1 April 2020)

\begin{abstract}
A Bose gas under the effect of Raman spin-orbit coupling (SOC) is analyzed using the discrete spin T-moves diffusion Monte Carlo method. Upon computing the energy as well as the static structure factor and the superfluid fraction of the system, the emergence of an energetically favorable supersolid stripe state is observed, which is in agreement with recent observations. A significant enhancement of the stability of the stripe phase with respect to the mean-field prediction is observed when the strength of the interatomic correlations is increased. We also quantify and characterize the degree of superfluidity of the stripes and show that this quantity is mostly determined by the ratio between the Raman coupling and the square of the momentum difference between the pair of SOC-inducing laser beams.
\end{abstract}

DOI: 10.1103/PhysRevA.101.043602

\section{INTRODUCTION}

Spin-orbit coupling (SOC), which denotes the interplay between a particle's momentum and its spin, has been a subject of interest in recent years, both theoretically and experimentally. This is due to the wide variety of exotic quantum states induced by this kind of interaction, which include topological insulators [1], topological superconductors [2], and Majorana fermions [3]. SOC is a relativistic effect that emerges naturally in electronic systems and that is also synthetically engineered [4] in ultracold atomic gases. These recent realizations in dilute gases represent an important achievement in the study of the physics of SOC due to the high controllability and tunability of these systems. In the particular case of Raman SOC, its implementation was first achieved experimentally by inducing a Raman coupling via two laser beams on an atomic $\Lambda$-type configuration. SOC is then generated by the simultaneous driving of a spin-flip transition and transferring of momentum [5-9]. Under this scheme, Raman SOC has been realized with ${ }^{87} \mathrm{Rb}$ bosons, both in the continuum [5] and in a lattice [10,11], and also with other species: ${ }^{6} \mathrm{Li}[8],{ }^{40} \mathrm{~K}$ [7], ${ }^{87} \mathrm{Sr}[12],{ }^{173} \mathrm{Yb}[13,14]$, and ${ }^{161} \mathrm{Dy}[15]$. In this context, two hyperfine states of the atom are labeled spin states.

In this paper, we focus on Raman SOC, which couples the linear momentum of an atom with its spin according to

$$
\hat{W}^{\mathrm{SOC}}=\frac{\hbar k_{0}}{m} \hat{P}_{x} \hat{\sigma}_{z}+\frac{\hbar^{2} k_{0}^{2}}{2 m}-\frac{\Omega}{2} \hat{\sigma}_{x},
$$

with $m$ the mass of the particle, $\hat{P}_{x}$ the $x$ component of the momentum, $\hat{\sigma}_{x}$ and $\hat{\sigma}_{z}$ the Pauli matrices, $\Omega$ the Raman coupling, and $k_{0}$ the magnitude of the wave-vector difference between

\footnotetext{
*juan.sanchez.baena@upc.edu

†jordi.boronat@upc.edu

‡ferran.mazzanti@upc.edu
}

the two laser beams. Some striking features induced by the SOC interaction can be observed already at the single-particle level. The coupling between momentum and spin implies that the minimum of the energy dispersion relation is in a nonzeromomentum, degenerate state for a given range of values of the Raman coupling [16]. This degeneracy involves states of equal magnitude but opposite sign in momentum space, enabling the possibility of a stripe-phase ground state. The inclusion of interactions changes this behavior, and depending on the parameters of the Hamiltonian a single momentum state or a stripe state is favored, each one with a different momentum.

Supersolid stripes arise from the breaking of two symmetries: gauge symmetry, giving rise to off-diagonal longrange order; and spatial symmetry, seen as a periodic density modulation in space [17]. The emergence and characterization of stripes in SOC systems have been a subject of major relevance in the field, from both the theoretical $[17,18]$ and the experimental sides [19]. Despite being predicted by theory, the stripe phase was not detected in the first experimental realization of Raman SOC by Spielman's group [5], mainly due to the extremely low spin dependence of the interatomic interactions between ${ }^{87} \mathrm{Rb}$ atoms. Later, Ketterle's group [19] provided evidence of its existence through Bragg scattering, using a new Raman SOC setup with ${ }^{23} \mathrm{Na}$ that allowed for better control of the spin interactions. Recently, they have also been detected in another experiment with ${ }^{87} \mathrm{Rb}$ atoms [20], where the contrast of the stripes is enhanced by rapidly increasing the Raman coupling before probing the density modulations by optical Bragg scattering.

In previous theoretical works, the phase diagram of an interacting system of atoms under Raman SOC has been reported $[16,21]$. However, the diagram is restricted to the mean-field regime, valid only for very low gas parameter values, $\leqslant 10^{-4}$. In order to extend these results to the stronger interacting regime and to deal with the nonlocal character of the SOC interaction, we use the discrete spin T-moves diffusion Monte Carlo (DTDMC) method [22-24] to study 
the system from a microscopic point of view. Starting from a variational ansatz, we propagate the initial wave function in imaginary time keeping its phase constant, leading to a statistical representation of the best possible wave function given a phase constraint (fixed-phase approximation). The DTDMC is then used to sample relevant observables, some of which may not be easily calculated at the mean-field level. An exact form of the imaginary time propagator up to first order in the time step is employed.

This paper is organized as follows. In Sec. II we discuss the Hamiltonian of the system and the relevant parameters used in the elaboration of the phase diagram. In Sec. III we show and discuss the phase diagram of the system, as well as the static structure factor, the pair distribution function, and the superfluid fraction, focusing on the stripe phase. Finally, in Sec. IV we summarize the main conclusions of our work.

\section{HAMILTONIAN}

We study a three-dimensional system of $N$ bosons of mass $m$ under periodic boundary conditions described by the Hamiltonian

$$
\hat{H}=\sum_{i}\left[\frac{\hat{P}_{i}^{2}}{2 m}+\hat{W}_{i}^{\mathrm{SOC}}\right]+\sum_{i<j} \hat{V}_{i j},
$$

where $\hat{V}_{i j}$ is a short-range, two-body, spin-dependent interaction. We use two model interactions: a soft-sphere (SS) potential of strength $V_{0}\left(s_{i}, s_{j}\right)$ and range $R_{0}\left(s_{i}, s_{j}\right)$ and a Lennard-Jones (LJ) force $V_{i j}\left(r_{i j}, s_{i}, s_{j}\right)=\left(\frac{\sigma_{12}\left(s_{i}, s_{j}\right)}{r_{i j}}\right)^{12}-$ $\left(\frac{\sigma_{6}\left(s_{i}, s_{j}\right)}{r_{i j}}\right)^{6}$. Here, $r_{i j}$ is the distance between the $i$ th and the $j$ th particles, and $s_{i}, s_{j}= \pm 1$ are their spins. The trial wave function used for importance sampling in the DTDMC method, which also fixes the phase, is chosen as the product of one-body and two-body (Jastrow) terms. For the former, we use the expression reported in Ref. [16], with the sign of the spin-down component changed due to the different sign of the $\Omega$ term in the Hamiltonian. The Jastrow factor depends on the interaction $\hat{V}_{i j}$. For the SS potentials we use the zeroenergy solution of the averaged interaction along the different spin channels, which provides a lower variational energy than a spin-dependent two-body Jastrow factor. In the case of the LJ interaction a McMillan factor of the form $e^{-(b / r)^{5}}$ is used, with $b$ a constant that is variationally optimized. The choice of the parameters of the two-body interaction $\hat{V}_{i j}$ determines the different channel scattering lengths $a_{s, s^{\prime}}$, as according to Ref. [25] the inclusion or omission of the SOC term does not appreciably change them. The values used in this work fulfill the condition $a_{+1,+1}=a_{-1,-1}>a_{+1,-1}=a_{-1,+1}$, as in the experiments in Ref. [19]. Finally, we express all quantities in dimensionless form, introducing characteristic length $\left(a_{0}=\right.$ $\left.1 / k_{0}\right)$ and energy $\left(E_{0}=\hbar^{2} k_{0}^{2} / 2 m\right)$ scales.

In order to characterize the phase diagram of the model, we use the standard gas parameter $n a^{3}$, with $a=a_{+1,+1}$ the scattering length of the interaction in the $(+1,+1)$ channel. It should be noted that, for this system, $n a^{3}$ is not a scaling parameter. However, we use it to characterize the combined effect of the density and the interaction. We have checked, though, that for very low values of $n a^{3}$ one recovers the mean-field results, while for larger values, the DTDMC simulations reveal that the extension of the stripe-phase domain is increased with respect to the mean-field prediction. This may be a relevant issue for experiments aiming to detect and/or characterize the stripe phase. In order to illustrate this, we set the density to $n=3.7 \times 10^{-3}$, with the number of particles $N \in[50,120]$ and the size of the simulation box changing as a function of the momentum of the trial wave function. We tune the spin-dependent scattering lengths such that $n a^{3} \in$ $\left(10^{-4}, 10^{-1}\right)$ by changing the two-body potential parameters. In this sense, increasing the gas parameter is equivalent to increasing the range and strength of the interactions, which enhances the effect of correlations in the medium. We set the interaction contrast $\gamma=\left(a-a_{+1,-1}\right) /\left(a+a_{+1,-1}\right)$ to $\gamma=$ 0.4 , since nonzero values of this quantity are necessary for the existence of a stripe ground state [16]. The quantitative characterization of the superfluidity in the stripe phase is performed with the contrast used in Ref. [19], $\gamma=0.904$. It must be remarked that the quantity $\gamma$ is a tunable property in the experimental setup in Ref. [19].

\section{PHASE DIAGRAM AND OTHER OBSERVABLES}

The phase diagram of the SOC system is reported in Fig. 1 for a fixed density and varying scattering length. The upper and middle plots correspond to the DTDMC results for the SS and LJ interactions, respectively, while the lower plot shows the mean-field phase diagram. The points indicate the computed transition lines between the different phases. Error bars in the DTDMC results account for the statistical variance of the energy estimations. Looking at the DTDMC phase diagrams it can be seen that, as the two-body scattering length increases, the value of the reduced Raman coupling at which the plane-wave-stripe-phase transition takes place also increases. Remarkably, this effect is absent at the meanfield level and is also robust with respect to the interaction employed. Based on this, we conclude that the enhancement of the stripe phase in the DTDMC phase diagrams is produced by the increase in interatomic correlations. This enhancement takes place because the DTDMC correction to the energy of the stripe $\left(\Delta E_{\mathrm{DMC}, \mathrm{S}}\right)$ and plane-wave $\left(\Delta E_{\mathrm{DMC}, \mathrm{PW}}\right)$ phases and the energy difference between these phases at the mean-field level $\left(\Delta E_{\mathrm{MF}}\right)$ fulfill $\left|\frac{\Delta E_{\mathrm{DMC}, \mathrm{S}}}{\Delta E_{\mathrm{MF}}}\right| \simeq\left|\frac{\Delta E_{\mathrm{DMC}, \mathrm{PW}}}{\Delta E_{\mathrm{MF}}}\right| \simeq 1$ over a wide region of the phase diagram. The stripe phase is favored over the plane-wave phase in the DTDMC diagram because of the different polarization between phases: while the stripe phase is always unpolarized, the plane-wave phase has nonzero polarization. Since the potentials employed in this work are less repulsive in the $(+1,-1)$ and $(-1,+1)$ channels in accordance with the experiment in Ref. [19], the beyond-mean-field corrections favor an unpolarized state over a polarized one. In this sense, the DTDMC corrections drastically determine the transition line. In contrast, the single-minimum region of the diagram is only slightly changed by the DTDMC with respect to the mean-field prediction. This is because the energy gap in the mean field between this phase and the stripe and planewave phases is larger in absolute value than the DTDMC corrections over the majority of the phase diagram.

In the mean field, the stripe-plane-wave and the stripe-single-minimum transitions are of first order, while 

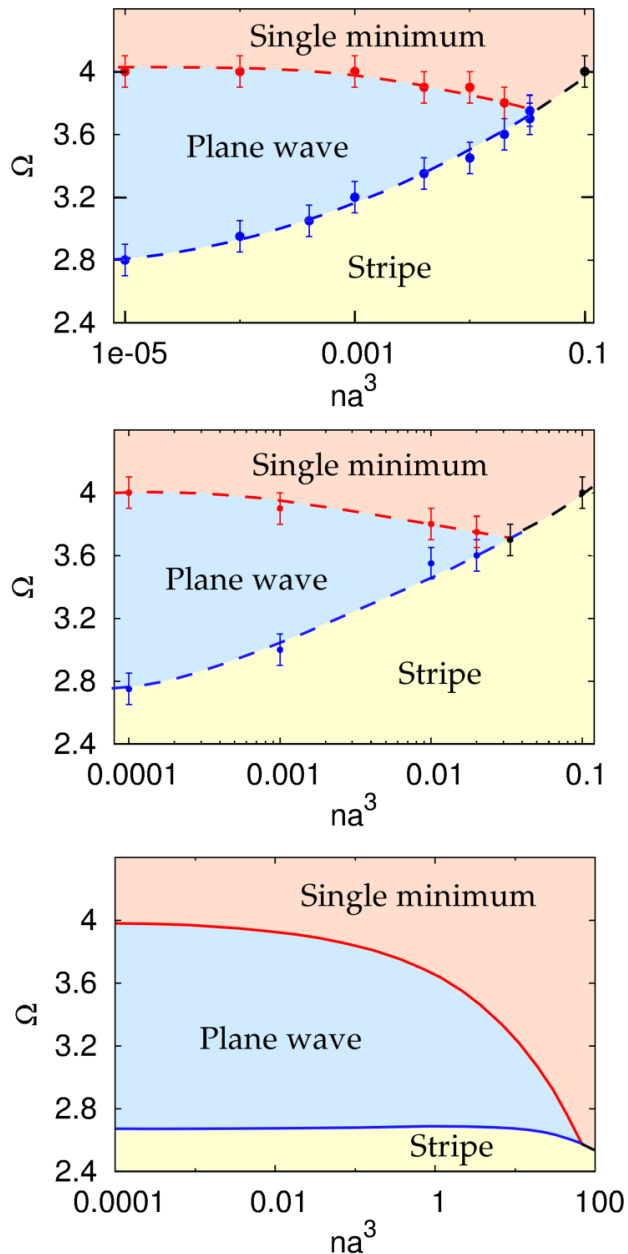

FIG. 1. Phase diagram of the many-body system with Raman spin-orbit coupling. The upper plot corresponds to the DTDMC diagram using the SS potential and the middle one to the LJ potential. In the lower plot, we report the mean-field phase diagram.

the plane-wave-single-minimum transition is of second order $[4,21]$. This is directly reflected in the value of the momentum that minimizes the energy in each phase at the mean-field level: while there is a discontinuity in this parameter between the stripe and the other two phases at the transition, the optimal momentum changes continuously from the planewave to the single-minimum phases $[4,21]$. We believe that the inclusion of correlations in the DTDMC calculation does not change the nature of any of these phase transitions.

The presence of interatomic correlations can be seen in the pair distribution function, $g\left(\vec{r}_{i}-\vec{r}_{j}\right)$, which yields the probability of finding two particles with relative position vector $\vec{r}_{i}-\vec{r}_{j}$. For an isotropic system, $g(\vec{r})$ depends on $|\vec{r}|$, while for a nonisotropic system, as in the case of the stripe phase, an expansion in partial waves of the form $g\left(\vec{r}_{i}-\vec{r}_{j}\right)=$ $\sum_{l, m} g_{l, m}\left(r_{i j}\right) Y_{l}^{m}(\theta, \phi)$ yields nonzero contributions for $l>0$. Note that, in this expression, $\theta$ is the angle formed by $\vec{r}$ and the $x$ axis, and stripes are formed along planes perpendicular to that direction. In Fig. 2, we show the leading correction to the isotropic mode, for two points in the phase diagrams corresponding to the stripe and plane-wave phases. Only the $(+1,+1)$ component is reported since the results for the rest

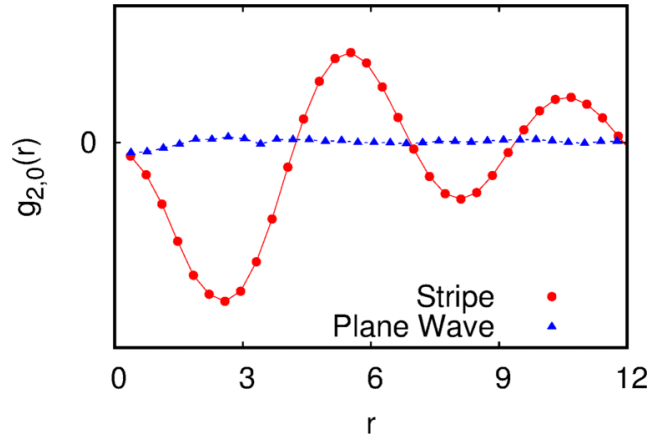

FIG. 2. Leading correction to the isotropic contribution to the pair distribution function in the $(+1,+1)$ channel, corresponding to $l=2, m=0$ for the SS interaction.

of the two-body channels are analogous. The figure depicts the $l=2, m=0$ modes for the SS interaction. It should be pointed out that, for the specific type of interactions used in this work, only the $m=0$ contributions survive. As one can see, $g_{2,0}\left(r_{i j}\right)$ is 0 in the plane-wave phase, while it yields a non-vanishing contribution in the stripe phase. This reflects the different spatial symmetries associated with each phase [16]. This quantity also vanishes for the single-minimum phase. Very similar results hold for the LJ interaction.

Since in the stripe phase the $x$ axis is transverse to the stripe planes, the static structure factor along the $x$ direction, $S\left(k_{x}\right)$, develops a peak at a momentum proportional to the inverse of the characteristic distance separating the stripes, a feature also present at the Bogoliubov level [17]. We show in the upper panel in Fig. 3 the static structure factor $S(\vec{k})$
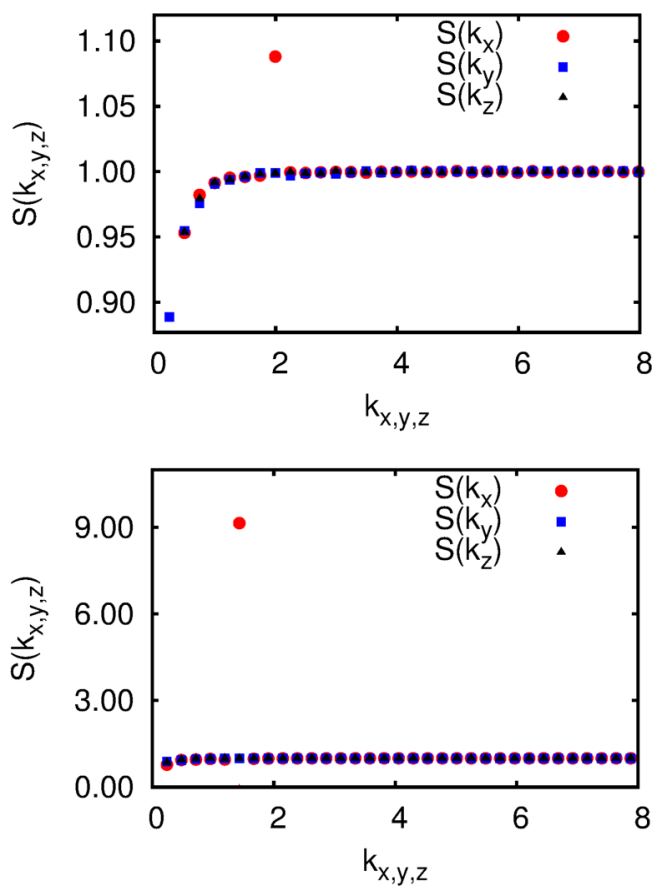

FIG. 3. Static structure factors for the SS interaction, for two different points with $n a^{3}=5 \times 10^{-5}$ and $\gamma=0.904$, both corresponding to the stripe phase. Upper panel, $\Omega=0.3131$; lower panel, $\Omega=2.8$. 


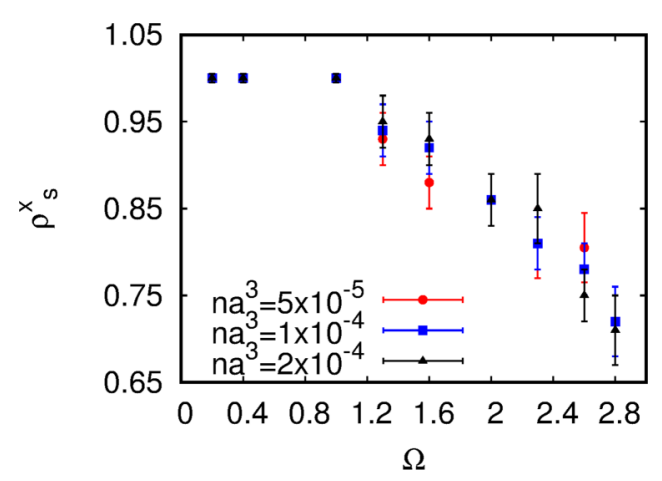

FIG. 4. Superfluid fraction for the transverse direction to the stripe planes as a function of the reduced Raman coupling for $n a^{3}=$ $5 \times 10^{-5}, \gamma=0.904$.

for conditions similar to those in the experiment in Ref. [19] $\left(\gamma=0.904, \Omega=0.3131\right.$, and $\left.n a^{3}=5 \times 10^{-5}\right)$. The lower panel shows the same quantities for $\Omega=2.8$, where the stripe modulation is much more important due to the higher value of $\Omega$ compared to $E_{0}$. In accordance with that experiment, where reduced Raman coupling values lie in the interval $\Omega_{\exp } \in$ $[0,0.4]$, we recover the stripe phase as the lowest-energy state. The periodicity of the stripes has been quantitatively characterized before both in the mean-field regime $[16,17]$ and in experiments [19]. The static structure factor does not show any peak in the plane-wave and single-minimum phases, a consequence of the lack of density modulations in these phases [16].

Finally, we characterize the superfluidity of the system in the stripe phase, where other systems have shown a nontrivial dependence along different directions [26]. In order to recreate the conditions of contrast and diluteness from Ref. [19], we set $\gamma=0.904$ and use gas parameters spanning the range $n a^{3} \in\left[5 \times 10^{-5}, 2 \times 10^{-4}\right]$. We measure the superfluid density using the zero-temperature limit of the winding number estimator [27], which is extracted from the mean squared displacement of the center of mass of the particles during imaginary time evolution. We show in Fig. 4 results for the superfluid fraction $\rho_{s}^{x}$ in the stripe phase along the $x$ direction, obtained from the generalization of the expression reported in Ref. [26], as a function of $\Omega$, and for three values of the gas parameter, $n a^{3}=5 \times 10^{-5}, 1 \times 10^{-4}$, and $2 \times 10^{-4}$. The reported values are close to those reported in Ref. [28], obtained at the mean-field level using the twisted-phase method. We see from the plot that the main parameter governing changes in $\rho_{s}^{x}$ is $\Omega$, while little dependence on the specific value of the gas parameter is found. As $\Omega$ increases, the system becomes less superfluid in the $x$ direction. This is a direct consequence of the fact that the amplitude of the density modulation increases with $\Omega$, as already seen in mean-field theory. For large values of $\Omega$, exchanges of particles between different stripe planes are less favored, and thus localization along the $x$ axis is enhanced. In the other two directions, parallel to the stripe planes, the system remains fully superfluid $\left(\rho_{s}^{y}=\rho_{s}^{z}=1\right)$.
Note also that, for the values of $\Omega$ employed in the experiment in Ref. [19], the superfluid fraction $\rho_{s}^{x}$ equals 1 . This, together with the periodic density modulations in the static structure factor reported in Fig. 3, yields a quantitative indication of simultaneous spatial periodicity and superfluidity in the system.

The superfluid fraction for the plane-wave and singleminimum phases has been obtained at the mean-field level using the phase twist method [28] and in the Bogoliubov model through the evaluation of the transverse current operator [29]. In this case, the superfluidity along the $y$ and $z$ axes in these phases is equal to unity, while $\rho_{x}^{s}$ shows a dependence on the Raman coupling. We recover these results with the DTDMC for the gas parameters mentioned previously by using the expression for the normal density from Ref. [29], replacing the mean-field value of $\left\langle\sigma_{x}\right\rangle$ with the one provided by the DTDMC.

\section{CONCLUSIONS}

In summary, we have shown, using the DTDMC method $[22,23]$, that by increasing the strength of interatomic correlations in a system under Raman SOC, the region of the phase diagram covered by the stripe phase is enlarged in comparison to the prediction of mean-field theory. We have shown that this effect holds for different two-body model interactions (soft spheres and Lennard-Jones), which provide very similar results. The breaking of continuous translational symmetry in the stripe phase has been characterized by the presence of a Bragg peak in the static structure factor and by a nonzero contribution to partial waves other than the $l=0$ to the pair distribution function. We have also performed DTDMC calculations under the same conditions of interaction contrast and reduced Raman coupling as for the experiments in Ref. [19]. Our results confirm the observed stripes as the most energetically favorable state and quantitatively show the supersolid behavior of the stripes. We have also studied the superfluid fraction of the stripe phase as a function of the reduced Raman coupling and the gas parameter by changing the scattering length of the interatomic interaction. We have shown that superfluidity in the stripe phase decreases mainly as a function of the reduced Raman coupling, with little dependence on the gas parameter in the range analyzed. We hope that our work can encourage possible experimental studies of Raman SOC systems near the transition lines between the stripe and the plane-wave phases, since the effects of correlations beyond the mean-field approximation can be seen even at relatively low gas parameter values like $n a^{3}=10^{-4}$.

\section{ACKNOWLEDGMENTS}

We acknowledge interesting discussions with S. Stringari on the superfluidity of the different SOC phases. This work was supported by MINECO (Spain) Grant No. FIS201784114-C2-1-P. J.S.-B. also acknowledges a FPU fellowship (reference FPU15/01805) from MCIU (Spain).
[1] M. Z. Hasan and C. L. Kane, Rev. Mod. Phys. 82, 3045 (2010).
[2] M. Sato and Y. Ando, Rep. Prog. Phys. 80, 076501 (2017).

[3] F. Wilczek, Nat. Phys. 5, 614 (2009). 
[4] L. Zhang and X. Liu, in Synthetic Spin-Orbit Coupling in Cold Atoms, edited by W. Zhang (World Scientific, Singapore, 2018), pp. 1-87.

[5] Y. J. Lin, K. Jiménez-García, and I. B. Spielman, Nature 471, 83 (2011).

[6] D. Zhang, T. Gao, P. Zou, L. Kong, R. Li, X. Shen, X. L. Chen, S. G. Peng, M. Zhan, H. Pu, and K. Jiang, Phys. Rev. Lett. 122, 110402 (2019).

[7] P. Wang, Z.-Q. Yu, Z. Fu, J. Miao, L. Huang, S. Chai, H. Zhai, and J. Zhang, Phys. Rev. Lett. 109, 095301 (2012).

[8] L. W. Cheuk, A. T. Sommer, Z. Hadzibabic, T. Yefsah, W. S. Bakr, and M. W. Zwierlein, Phys. Rev. Lett. 109, 095302 (2012).

[9] X.-J. Liu, M. F. Borunda, X. Liu, and J. Sinova, Phys. Rev. Lett. 102, 046402 (2009).

[10] C. Hamner, Y. Zhang, M. A. Khamehchi, M. J. Davis, and P. Engels, Phys. Rev. Lett. 114, 070401 (2015).

[11] T. M. Bersano, J. Hou, S. Mossman, V. Gokhroo, X. W. Luo, K. Sun, C. Zhang, and P. Engels, Phys. Rev. A 99, 051602(R) (2019).

[12] S. Kolkowitz, S. L. Bromley, T. Bothwell, M. L. Wall, G. E. Marti, A. P. Koller, X. Zhang, A. M. Rey, and J. Ye, Nature 542, 66 (2017).

[13] B. Song, C. He, S. Zhang, E. Hajiyev, W. Huang, X.-J. Liu, and G.-B. Jo, Phys. Rev. A 94, 061604(R) (2016).

[14] L. F. Livi, G. Cappellini, M. Diem, L. Franchi, C. Clivati, M. Frittelli, F. Levi, D. Calonico, J. Catani, M. Inguscio, and L. Fallani, Phys. Rev. Lett. 117, 220401 (2016).

[15] N. Q. Burdick, Y. Tang, and B. L. Lev, Phys. Rev. X 6, 031022 (2016).
[16] Y. Li, G. I. Martone, and S. Stringari, Annual Review of Cold Atoms and Molecules, Vol. 3 (World Scientific, Singapore, 2015), pp. 201-250.

[17] Y. Li, G. I. Martone, L. P. Pitaevskii, and S. Stringari, Phys. Rev. Lett. 110, 235302 (2013).

[18] W. Han, G. Jazeliunas, W. Zhang, and W. M. Liu, Phys. Rev. A 91, 013607 (2015).

[19] J. Li, J. Lee, W. Huang, S. Burchesky, B. Shteynas, F. Ç. Top, A. O. Jamison, and W. Ketterle, Nature 543, 91 (2017).

[20] A. Putra, F. Salces-Cárcoba, Y. Yue, S. Sugawa, and I. B. Spielman, Phys. Rev. Lett. 124, 053605 (2020).

[21] Y. Li, L. P. Pitaevskii, and S. Stringari, Phys. Rev. Lett. 108, 225301 (2012).

[22] J. Sánchez-Baena and J. Boronat, and F. Mazzanti, Phys. Rev. A 98, 053632 (2018).

[23] C. Melton, M. Chandler Bennett, and L. Mitas, J. Chem. Phys. 144, 244113 (2016).

[24] M. Casula, S. Moroni, C. Filippi, and S. Sorella, J. Chem. Phys. 132, 154113 (2010).

[25] X. Cui, Phys. Rev. A 85, 022705 (2012).

[26] R. Bombin, J. Boronat, and F. Mazzanti, Phys. Rev. Lett. 119, 250402 (2017).

[27] S. Zhang, N. Kawashima, J. Carlson, and J. E. Gubernatis, Phys. Rev. Lett. 74, 1500 (1995).

[28] X. L. Chen, J. Wang, Y. Li, X. J. Liu, and H. Hu, Phys. Rev. A 98, 013614 (2018).

[29] Y. Zhang, Z. Yu, T. K. Ng, S. Zhang, L. Pitaevskii, and S. Stringari, Phys. Rev. A 94, 033635 (2016). 